\title{
Shared Pheromonal Communication of Specific Fear Between Adult Sprague Dawley Rats
}

\author{
Samantha J. Carew* and Abhinaba Ghosh
}

BioMedical Sciences, Memorial University of Newfoundland, St. John's, Canada

*For correspondence: scarew@mun.ca

[Abstract] Rats are highly social animals, and mainly communicate with one another in two ways: through ultrasonic vocalizations and pheromones. Most research on pheromones has been dedicated those regarding sexual behavior, but more recently pheromones which signal danger to conspecifics have been identified in rodents. In fact, rats are capable of communicating information regarding a specific fear to a companion with which they share a cage. If a rat is trained to associate a previously neutral odor with a foot shock and then pair housed with another rat, the companion will also display a fear response specific to the trained odor, despite never being shocked itself. This communication relies on pheromones; presenting soiled bedding from a shocked rat to an individually housed naïve rat produces the same fear response in the naïve rat. The current protocol describes how to produce this phenomenon in adult Sprague Dawley rats. It is simple and easily reproduced, requires minimal equipment, and may be completed within one week.

Keywords: Alarm Pheromone, Conditioning, Fear, Shock, Pair-housed, Freezing, Olfaction, Odor

[Background] Like humans, rats are considered a social species; they tend to thrive with conspecifics rather than in isolation. Generally, rats can relay information through ultrasonic vocalizations and pheromones. Given their highly developed olfactory systems, a wide array of information may be conveyed through the sense of smell. Releasing and receiving pheromonal signals play critical roles in both neurobiological and ethological aspects of rodent behaviour. These include but are not limited to the selection of mating partners (Kumar et al., 2014), spreading alarm signals (Kiyokawa et al., 2013), novel food acquisition (Posadas-Andrews and Roper, 1983), territory marking (Brain et al., 2009), demonstrating social status (Krames et al., 1969), and maternal interaction (Bind et al., 2013). Pheromonal signaling can actually interfere with several biological functions in a laboratory setting; the presence of unfamiliar males can prevent pregnancy (Bruce, 1959), female estrous cycles may become prolonged when exposed to urine from a lactating female (Hoover and Drickamer, 1979), and male urine can cause acceleration of puberty in females (Vandenbergh, 1975). It has been known for some time that certain pheromones can carry information regarding sexual behaviours (Hayashi and Kimura, 1974; Gawienowski et al., 1975), but more recently it has been conclusively shown that mammals can also communicate information about stress via alarm pheromones (Inagaki et al., 2014; Kiyokawa, 2017).

Social fear learning is a growing field in neuroscience and many models have been used to attempt to study how it works. The subtle differences in approach give clues as to how rodents are actually able to communicate with one another. Knapska et al. (2006) exposed rats to shock in a novel context then allowed their cagemate, the "observer", to interact with this "demonstrator" rat. They found that observer 
rats housed with a demonstrator that had been fear conditioned spent more time interacting with the demonstrator and an enhanced acoustic startle response following this interaction, suggesting a transfer of emotional states between the two animals. Bruchey et al. (2010) developed a fear conditioning by proxy paradigm whereby a naïve rat watches its cagemate respond to a previously fear conditioned auditory stimulus. When the trained cagemate freezes in response to the tones, this causes the naïve rat to develop a fear reaction to subsequent presentations of the conditioned stimulus. Although the naïve rat had never received a shock, witnessing fearful behavior of its cagemate in response to the tone was enough to cause a conditioned response (albeit weaker than the classically conditioned fear) in some of the rats. The degree of success in fear conditioning by proxy was correlated with the duration of social interaction during the procedure. Again, it is clear that interaction with a stressed conspecific is sufficient to induce some behavioural change in a rat's emotional response.

Since rats rely so heavily on their sense of smell, it is likely that this communication occurs through an olfactory mode, likely via pheromones, as opposed to ultrasonic vocalizations. Debiec and Sullivan (2014) found that a stressed mother's scent delivered through an olfactometer paired with a novel odor caused avoidance to that odor in pups. A series of experiments from the Mori group has identified a rat alarm pheromone that causes stress-induced hyperthermia in recipient rats, as well as increased defensive and risk assessment behaviours combined with a decrease in exploratory and grooming behavior (Kikusui et al., 2001; Kiyokawa et al., 2006). This rat alarm pheromone has been isolated and two of its components, 4-methylpentanal and hexanal, produce the same behavioural effects as the pheromone itself (Inagaki et al., 2014). Two things are clear from the previous research: 1) information can be conveyed regarding emotional responses between conspecific rats and 2) interaction between a naïve rat and either a conditioned rat or a pheromonal component of its odor is important for this communication.

The current protocol describes the use of same sex 8-10-week old Sprague Dawley rat pairs as odor + shock $\left(\mathrm{O}^{+} / \mathrm{S}^{+}\right)$and companion $\left(\mathrm{O}^{+} / \mathrm{Comp}\right)$ conditions in a paradigm by which a conditioned fear of a specific odor is communicated from the $\mathrm{O}^{+} / \mathrm{S}^{+}$rat to its companion. This paradigm produces a socially conditioned fear in a rat whose cagemate has been classically conditioned to associate an odor with a shock. Unshocked $\mathrm{O}^{+} / \mathrm{SB}$ rats are also able to acquire this fear in the absence of a cage mate by being exposed to the soiled bedding of an $\mathrm{O}^{+} / \mathrm{S}^{+}$rat along with the conditioned odor. In fact, pairing previously identified alarm pheromone components with the conditioned odor also produces this phenomenon in unshocked $\mathrm{O}^{+} / \mathrm{Ph}$ rats. Together, these experiments provide strong support to the notion that rats are able to communicate a specific fear through pheromones. As opposed to other methodologies, the current protocol may reflect actual communication through pheromones rather than just a transfer of emotional states, as the socially acquired fear is specific to the trained odor and is not generalized to a different stimulus if presented after the interaction. The protocol described below does not require expensive equipment or materials, and can be completed within one week. 


\section{Materials and Reagents}

1. Gloves

2. Labcoat

3. Hairnet

4. Face mask

5. Rats

6. a-Terpinene (Millipore Sigma, Sigma-Aldrich, catalog number: 86473), store in flammable cabinet

7. Octanol (Millipore Sigma, Sigma-Aldrich, catalog number: 297887), store in flammable cabinet

8. Mineral Oil (Millipore Sigma, Sigma-Aldrich, catalog number: 330760), store in flammable cabinet

9. 4-methylpentanal (Santa Cruz Biotechnology, catalog number: sc-483809), store in flammable cabinet

10. Hexanal (Santa Cruz Biotechnology, catalog number: sc-252885), store in flammable cabinet

11. $100 \%$ ethanol (Commercial Alcohols, catalog number: P016EAAN), store in flammable cabinet

12. Purified water

13. Terpinene (6.63\%) (see Recipes)

14. Octanol (2.67\%) (see Recipes)

15. 4-methylpentanal $\left(1.6 \times 10^{-6} \mathrm{M}\right)$ and hexanal $\left(8.7 \times 10^{-6} \mathrm{M}\right)$ binary mixture (see Recipes)

16. Ethanol (70\%) (see Recipes)

\section{Equipment}

1. Shock generator with grid and removable plexiglass tray (Muromachi, Kikai, model: SGS-003DX)

2. Plexiglass box without bottom (Custom made)

3. Clear lid for plexiglass box (Custom made)

4. Fan-attached lid for plexiglass box (Custom made)

Note: The fan blows odors out of box through evacuation tubing which is connected to ventilation system in the ceiling. This allows for minimum permeation of odors into the room.

5. Evacuation tubing

6. Odor delivery system (Custom made)

7. Odor delivery bottles (Polyvinyl carbonate bottles with 2 holes in lid for c-flex tubing)

8. Odor delivery c-flex tubing

9. Medical air tank $\left(95 \% \mathrm{O}_{2} 5 \% \mathrm{CO}_{2}\right)$

Note: See Figure 1 for a labeled image of the experimental setup.

10. Air exchanger

11. Timer

12. Stopwatch 
13. Webcam and tripod

14. Laptop

15. Home cages

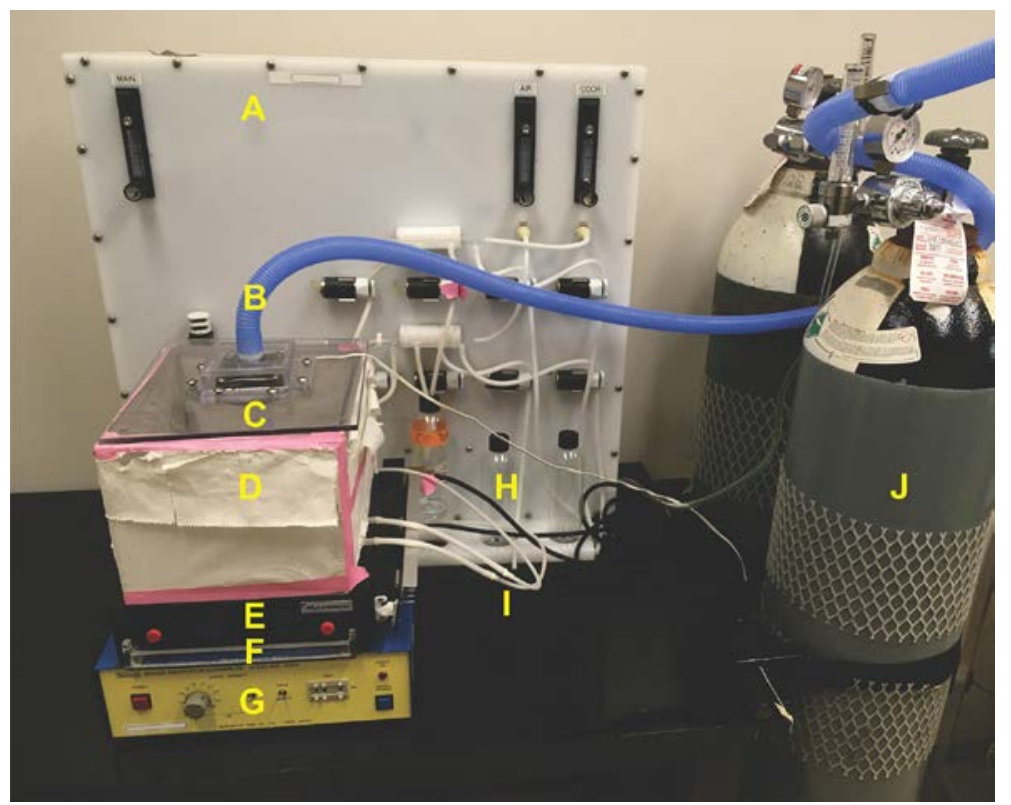

Figure 1. Labeled photograph of the custom-made experimental setup. A. Odor delivery system; B. Evacuation tubing; C. Fan-attached lid; D. Plexiglass box without bottom; E. Shock grid; F. Removable plexiglass tray; G. Shock generator; H. Odor delivery bottles; I. Odor delivery tubing (white); J. Medical air tank.

\section{Procedure}

A. Habituation

1. Complete during light cycle.

2. Keep rats on cart outside training room in home cages and allow rats to remain undisturbed for 20 min following transportation from animal care room prior to any behavioural procedure.

3. Turn on air exchanger.

4. Turn on medical air tank so air is freely flowing through shock box.

5. Wearing gloves, a labcoat, hairnet, and face mask, take rats one at a time from home cage, bring into training room, place in shock box (grid turned off) with fan-attached lid on top and turned on.

a. Rats in odor + shock $\left(\mathrm{O}^{+} / \mathrm{S}^{+}\right)$and odor only companion $\left(\mathrm{O}^{+} / \mathrm{Comp}\right)$ conditions are pair housed starting a week prior to habituation.

b. Rats in odor only $\left(\mathrm{O}^{+} / \mathrm{S}^{-}\right)$, shock only $\left(\mathrm{O}^{-} / \mathrm{S}^{+}\right)$, and soiled bedding $\left(\mathrm{O}^{+} / \mathrm{SB}\right)$ conditions, and $\mathrm{O}^{+} / \mathrm{S}^{+}$soiled bedding donor rats are housed individually.

6. Let animal explore context for $30 \mathrm{~min}$.

7. Return animal to home cage. 
8. Clean the shock box.

a. Spray all interior surfaces with $70 \%$ ethanol (Recipe 4).

b. Wipe with paper towel.

c. Attach the lid with fan and evacuation tubing and turn on to dissipate odors.

d. Wait at least 10 min before moving on to next animal to ensure no residual odors remain in room.

9. Habituate each animal to shock box for 2 days, 30 min per day, on the 2 days prior to odor + shock conditioning.

This prevents the animal from associating the shock with the context, and promotes specific fear conditioning to the presented odor.

B. Training

1. Complete during light cycle.

2. Keep rats on cart outside training room in home cages and allow rats to remain undisturbed for 20 min following transportation from animal care room prior to any behavioural procedure.

3. Turn on air exchanger.

4. Turn on medical air flow.

5. Train animals for 1 day according to their condition, detailed below.

Note: Training of rats in condition c must always immediately precede conditions $d$ and e in cagemate/pairwise fashion but order of training for other groups does not matter.

a. $\mathrm{O}^{+} / \mathrm{S}$ Condition

i. Bring each rat to conditioning room and place in shock box (grid turned on) and cover with fan-attached lid turned on.

ii. Start timer.

iii. At 4 min, release c-flex tubing so terpinene odor enters shock chamber.

iv. Allow terpinene (Recipe 1) to flow through tubing for $1 \mathrm{~min}$, then close c-flex tubing to stop terpinene flow.

v. Repeat odor delivery as described in Steps B5aiii and B5aiv at 14, 19, and $29 \mathrm{~min}$.

vi. Remove rat from shock chamber and return to home cage immediately.

vii. Clean shock chamber.

1) Spray down all interior surfaces with $70 \%$ ethanol.

2) Place fan-attached lid on shock chamber and turn on to remove all residual odor.

3) Wait at least $10 \mathrm{~min}$ to introduce the next rat.

b. $\mathrm{O} / \mathrm{S}^{+}$Condition

i. Bring each rat to conditioning room and place in shock box (grid turned on) and cover with fan-attached lid turned on.

ii. Start timer.

iii. At 5, 15, 20, and 30 min press manual shock button to deliver one $1 \mathrm{~s} 0.5 \mathrm{~mA}$ shock.

iv. Remove rat from shock chamber and return to home cage immediately. 
v. Clean shock chamber.

1) Spray down all interior surfaces with $70 \%$ ethanol.

2) Place fan-attached lid on shock chamber and turn on to remove all residual odor.

3) Wait at least 10 min to introduce the next rat.

c. $\mathrm{O}^{+} / \mathrm{S}^{+}$Condition

i. Bring rat to conditioning room and place in shock box (grid turned on) and cover with fan-attached lid turned on.

Note: It is important that $\mathrm{O}^{+} /$Comp companion rat stays in home cage away from training room during $\mathrm{O}^{+} / \mathrm{S}^{+}$training.

ii. Start timer.

iii. At 4 min, release c-flex tubing so terpinene odor enters shock chamber.

iv. Allow terpinene to flow through tubing for $1 \mathrm{~min}$.

v. At 4:59 (during last second of odor delivery), press manual shock button to deliver one 1 s 0.5 mA shock.

vi. Close c-flex tubing to stop terpinene flow.

vii. Repeat odor delivery as described in Steps B5ciii-B5cvi at 14, 19, and $29 \mathrm{~min}$.

viii. Remove rat from shock chamber and return to home cage with companion rat immediately.

ix. Clean shock chamber.

1) Spray down all interior surfaces with $70 \%$ ethanol.

2) Place fan-attached lid on shock chamber and turn on to remove all residual odor.

3) Wait 10 min to introduce the next rat.

Note: This must be the corresponding companion rat ( $\left.\mathrm{O}^{+} / \mathrm{Comp}\right)$.

d. $\mathrm{O}^{+} /$Comp Condition

i. Following 10 min of interaction with freshly trained $\mathrm{O}^{+} / \mathrm{S}^{+}$companion rat, bring each rat to conditioning room and place in shock box (grid turned on) and cover with fan-attached lid turned on.

Note: It is important that $\mathrm{O}^{+} / \mathrm{S}^{+}$companion rat stays in home cage away from training room during $\mathrm{O}^{+} /$Comp training.

ii. Start timer.

iii. At 4 min, release c-flex tubing so terpinene odor enters shock chamber.

iv. Allow terpinene to flow through tubing for $1 \mathrm{~min}$, then close c-flex tubing to stop terpinene flow.

v. Repeat odor delivery as described in Steps B5diii and B5div at 14, 19, and 29 min.

vi. Remove rat from shock chamber and return to home cage with $\mathrm{O}^{+} / \mathrm{S}^{+}$companion rat immediately.

vii. Clean shock chamber.

1) Spray down all interior surfaces with $70 \%$ ethanol.

2) Place fan-attached lid on shock chamber and turn on to remove all residual odor. 
3) Wait at least $10 \mathrm{~min}$ to introduce the next rat.

e. $\mathrm{O}^{+} / \mathrm{SB}$ condition

Note: Train $\mathrm{O}^{+} / \mathrm{SB}$ rats immediately following training of separately singly housed $\mathrm{O}^{+} / \mathrm{S}^{+}$ soiled bedding donor rat, place bedding in tray underneath shock grid during $\mathrm{O}^{+} / \mathrm{S}^{+}$soiled bedding donor rat training, and do not clean shock box between animals, so that $O^{+} / S B$ is exposed to the soiled bedding of the $\mathrm{O}^{+} / \mathrm{S}^{+}$donor rat.

i. Bring each rat to conditioning room and place in shock box (grid turned on) and cover with fan-attached lid turned on.

ii. Start timer.

iii. At $4 \mathrm{~min}$, release c-flex tubing so terpinene odor enters shock chamber.

iv. Allow terpinene to flow through tubing for $1 \mathrm{~min}$, then close c-flex tubing to stop terpinene flow.

v. Repeat odor delivery as described in Steps B5eiii and B5eiv at 14, 19, and 29 min.

vi. Remove rat from shock chamber and return to home cage immediately.

vii. Clean shock chamber.

1) Spray down all interior surfaces with $70 \%$ ethanol.

2) Place fan-attached lid on shock chamber and turn on to remove all residual odor.

3) Wait at least 10 min to introduce the next rat.

f. $\mathrm{O}^{+} / \mathrm{Ph}$ condition

i. In tray underneath shock grid, place clean bedding material and a piece of filter paper soaked with $0.75 \mathrm{ml}$ 4-methylpentanal and hexanal binary mixture (Recipe 3).

ii. Bring each rat to conditioning room and place in shock box (grid turned on) and cover with fan-attached lid turned on.

iii. Start timer.

iv. At 4 min, release c-flex tubing so terpinene odor enters shock chamber.

v. Allow terpinene to flow through tubing for $1 \mathrm{~min}$, then close c-flex tubing to stop terpinene flow.

vi. Repeat odor delivery as described in Steps B5fiii and B5fiv at 14, 19, and 29 min.

vii. Remove rat from shock chamber and return to home cage immediately.

viii. Clean shock chamber.

1) Spray down all interior surfaces with $70 \%$ ethanol.

2) Place fan-attached lid on shock chamber and turn on to remove all residual odor.

3) Wait at least $10 \mathrm{~min}$ to introduce the next rat.

4) Replace bedding and filter paper soaked with $0.75 \mathrm{ml}$ 4-methylpentanal and hexanal binary mixture.

C. Testing

1. Complete during light cycle.

2. Keep rats on cart outside training room in home cages and allow rats to remain undisturbed for 
20 min following transportation from animal care room prior to any behavioural procedure.

3. Turn on the air exchanger.

4. Set up a laptop and webcam so rat is visible and testing session can be recorded.

5. Turn on medical air flow.

6. Wearing gloves, a labcoat, hairnet, and face mask, take rats one at a time from home cage, bring into training room, place in shock chamber (grid turned off) with clear lid on.

7. Start the timer.

8. Start recording video.

9. Keep medical air flowing through for $\mathbf{1 5}$ min to observe baseline behavior.

10. At $15 \mathrm{~min}$, release c-flex tubing so terpinene can flow through shock chamber.

11. Observe freezing behavior for 15 min during odor exposure.

Use stopwatch to record total amount of freezing time.

Note: This may be cut down to 5 min of medical air and 5 min of odor exposure.

12. Remove the rat from shock chamber and return to home cage.

13. Clean shock chamber.
a. Spray down all interior surfaces with $70 \%$ ethanol.
b. Place fan-attached lid on shock chamber and turn on to remove all residual odor.
c. Wait at least 10 min to introduce the next rat.

14. Repeat entire testing procedure using octanol (Recipe 2) (control odor) in Step C10 instead of terpinene in separate testing sessions during a different part of the day (e.g., if testing with terpinene was done in the morning, do octanol testing in the afternoon).

\section{Data analysis}

"Freezing" behaviour is considered the absence of all movements except for those required for respiration. To count time as positive freezing, the rat must be completely immobilized except for breathing. A bird's eye view (where the camera is placed) will show the rat's head low to the ground but not tucked into the body and its ears will be pulled back and flat to the skull. A stopwatch is used to record total time spent immobile and is started immediately upon observed freezing behavior and stopped immediately when rat movement is detected.

The percentage of freezing time per total time is compared among conditions using a one way analysis of variance (ANOVA) with a Bonferonni post-hoc test. Ideally at least $n=6$ would be included for data analysis.

Very limited movement during medical air and octanol exposure would be considered criteria for data exclusion, as well as any \% freezing score that falls outside of two standard deviations above or below the mean of the group. 
Freezing Time in Response to Odor Exposure

A

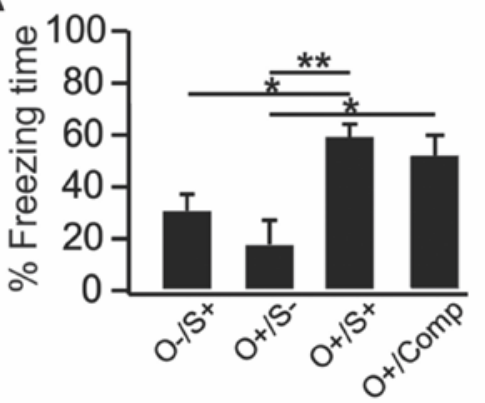

B

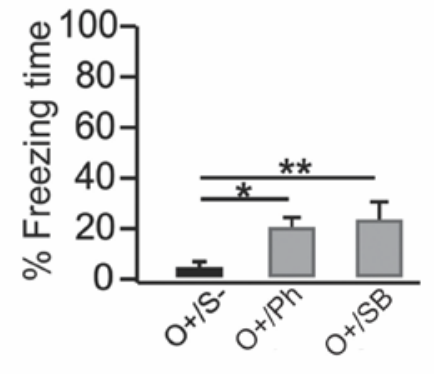

Figure 2. Freezing time as a percentage of the total time during a five-minute test exposure to the trained odor. A. Paired housing experiment. $\mathrm{O}^{-} / \mathrm{S}^{+}$, shock only rats; $\mathrm{O}^{+} / \mathrm{S}^{-}$, odor only rats that were caged alone; $\mathrm{O}^{+} / \mathrm{S}^{+}$, odor/shock conditioned rats; $\mathrm{O}^{+} / \mathrm{Comp}$, odor only rats that were caged with odor/shock conditioned rats. B. Single housing experiment. $\mathrm{O}^{+} / \mathrm{S}^{-}$, odor only rats; $\mathrm{O}^{+} / \mathrm{Ph}$, odor exposed rats that were conditioned with previously identified alarm pheromone molecules; $\mathrm{O}^{+} / \mathrm{SB}$, odor exposed rats that were conditioned with soiled bedding. Figure adapted from Carew et al., 2018.

\section{$\underline{\text { Notes }}$}

1. Ensure that other rats are far away from the rat currently being trained to rule out communication via ultrasonic vocalizations which may or may not occur during shock conditioning.

2. Ensure that all odors are removed reliably from the training room between rats.

3. Other odors may be used, but a preliminary test must ensure that the odors are not innately aversive or appetitive prior to training. An example of such a test would be a Y-maze, where an odor in question is used in one arm and its vehicle (mineral oil) in the other and time spent in each arm is recorded. If an odor is innately appetitive the rat will approach it greater than $50 \%$ of the time and if an odor is innately aversive the rat will avoid it more than $50 \%$ of the time, relative to mineral oil. We chose the odors terpinene and octanol based on their previously determined neutrality (Lee et al., 2013).

\section{$\underline{\text { Recipes }}$}

Note: Terpinene and octanol concentrations were chosen so that the odors would emit a vaporphase partial pressure of 1 Pascal (Lee et al., 2013).

1. Terpinene (6.63\%)
a. Add $1.326 \mathrm{ml}$ of $\alpha$-Terpinene to $18.67 \mathrm{ml}$ of mineral oil in a polyvinyl carbonate bottle
b. Final volume $20 \mathrm{ml}$
c. Vortex well to mix

2. Octanol (2.67\%) 

a. Add $534 \mu \mathrm{l}$ of 1 -Octanol to $19.47 \mathrm{ml}$ of mineral oil in a polyvinyl carbonate bottle
b. Final volume $20 \mathrm{ml}$
c. Vortex well to mix

3. 4-methylpentanal $\left(1.6 \times 10^{-6} \mathrm{M}\right)$ and hexanal $\left(8.7 \times 10^{-6} \mathrm{M}\right)$ binary mixture (Inagaki et al., 2014)
a. Add $16 \mu \mathrm{l}$ 4-methylpentanal and $87 \mu \mathrm{l}$ hexanal to $99.9 \mathrm{ml}$ of purified water
b. Final volume $100 \mathrm{ml}$
c. Vortex well to mix

4. Ethanol (70\%)
a. Add $350 \mathrm{ml}$ of $100 \%$ ethanol to $150 \mathrm{ml}$ deionized water in spray bottle
b. Final volume $500 \mathrm{ml}$

\section{Acknowledgments}

This protocol was derived from the below research paper: Carew et al. (2018).

\section{Competing interests}

The authors declare no competing interests.

\section{Ethics}

All procedures were approved by the Memorial University Institutional Animal Care Committee and carried out in compliance with the guidelines of the Canadian Council on Animal Care. Approval ID: 15-04-QY; Validity period: Feb 2016-Feb 2019.

\section{$\underline{\text { References }}$}

1. Bind, R. H., Minney, S. M., Rosenfeld, S. and Hallock, R. M. (2013). The role of pheromonal responses in rodent behavior: future directions for the development of laboratory protocols. $J$ Am Assoc Lab Anim Sci 52(2): 124-129.

2. Brain, P. F., Homady, M. H., Castano, D. and Parmigiani, S. (2009). Pheromones and behaviour of rodents and primates. Bollettino di Zoologia 54(4): 279-288.

3. Bruce, H. M. (1959). An exteroceptive block to pregnancy in the mouse. Nature 184: 105.

4. Bruchey, A. K., Jones, C. E. and Monfils, M. H. (2010). Fear conditioning by-proxy: social transmission of fear during memory retrieval. Behav Brain Res 214(1): 80-84.

5. Carew, S. J., Mukherjee, B., MacIntyre, I. T. K., Ghosh, A., Li, S., Kirouac, G. J., Harley, C. W. and Yuan, Q. (2018). Pheromone-induced odor associative fear learning in rats. Sci Rep 8(1): 17701. 
6. Debiec, J. and Sullivan, R. M. (2014). Intergenerational transmission of emotional trauma through amygdala-dependent mother-to-infant transfer of specific fear. Proc Natl Acad Sci U S A 111(33): 12222-12227.

7. Gawienowski, A. M., Orsulak, P. J., Stacewicz-Sapuntzakis, M. and Joseph, B. M. (1975). Presence of sex pheromone in preputial glands of male rats. J Endocrinol 67(2): 283-288.

8. Hayashi, S. and Kimura, T. (1974). Sex-attractant emitted by female mice. Physiol Behav 13(4): 563-567.

9. Hoover, J. E. and Drickamer, L. C. (1979). Effects of urine from pregnant and lactating female house mice on oestrous cycles of adult females. J Reprod Fertil 55(2): 297-301.

10. Inagaki, H., Kiyokawa, Y., Tamogami, S., Watanabe, H., Takeuchi, Y. and Mori, Y. (2014). Identification of a pheromone that increases anxiety in rats. Proc Natl Acad Sci U S A 111(52): 18751-18756.

11. Kikusui, T., Takigami, S., Takeuchi, Y. and Mori, Y. (2001). Alarm pheromone enhances stressinduced hyperthermia in rats. Physiol Behav 72(1-2): 45-50.

12. Kiyokawa, Y. (2017). Social odors: alarm pheromones and social buffering. Curr Top Behav Neurosci 30: 47-65.

13. Kiyokawa, Y., Kodama, Y., Kubota, T., Takeuchi, Y. and Mori, Y. (2013). Alarm pheromone is detected by the vomeronasal organ in male rats. Chem Senses 38(8): 661-668.

14. Kiyokawa, Y., Shimozuru, M., Kikusui, T., Takeuchi, Y. and Mori, Y. (2006). Alarm pheromone increases defensive and risk assessment behaviors in male rats. Physiol Behav 87(2): 383-387.

15. Knapska, E., Nikolaev, E., Boguszewski, P., Walasek, G., Blaszczyk, J., Kaczmarek, L. and Werka, T. (2006). Between-subject transfer of emotional information evokes specific pattern of amygdala activation. Proc Natl Acad Sci U S A 103(10): 3858-3862.

16. Krames, L., Carr, W. J. and Bergman, B. (1969). A pheromone associated with social dominance among male rats. Psychon Sci 16(1): 11-12.

17. Kumar, V., Vasudevan, A., Soh, L. J., Le Min, C., Vyas, A., Zewail-Foote, M. and Guarraci, F. A. (2014). Sexual attractiveness in male rats is associated with greater concentration of major urinary proteins. Biol Reprod 91(6): 150.

18. Lee, J., Linster, C., Devore, S., (2013). Odor preferences shape discrimination learning in rats. Behav Neurosci 127(4): 498-504.

19. Posadas-Andrews, A. and Roper, T. J. (1983). Social transmission of food-preferences in adult rats. Animal Behaviour 31(1): 265-271.

20. Vandenbergh, J. G., Whitsett, J. M. and Lombardi, J. R. (1975). Partial isolation of a pheromone accelerating puberty in female mice. J Reprod Fertil 43(3): 515-523. 